\title{
SECAGEM DE PASTAS EM LEITO DE JORRO: INFLUÊNCIA DAS CONDIÇÕES OPERACIONAIS
}

\author{
M. T. B. PERAZZINI, F. B. FREIRE e J. T. FREIRE
}

Universidade Federal de São Carlos, Departamento de Engenharia Química, Centro de Secagem E-mail para contato: freire@ufscar.br

\begin{abstract}
RESUMO - Neste trabalho foi realizado um estudo sobre o efeito do ângulo do cone e da massa de material inerte tanto na fluidodinâmica do leito seco, quanto na secagem de pastas em leito de jorro. O procedimento experimental foi conduzido utilizando um leito de jorro com coluna cilíndrica de $120 \mathrm{~cm}$ de altura, $30 \mathrm{~cm}$ de diâmetro, diâmetro de entrada de $3 \mathrm{~cm}$ e ângulos da base cônica de 45, 60 e 75 . Como inertes foram utilizadas partículas de polietileno e como pastas água destilada e leite desnatado. A massa de partículas inertes utilizada foi fixada em 1,5; 3,0 e 4,5kg para todos os valores de ângulo de cone estudados. As demais condições de operação foram $100^{\circ} \mathrm{C}$ e $1,3 \mathrm{u}_{\mathrm{mj}}$. Os resultados experimentais obtidos mostraram que a massa de material inerte apresentou efeito mais significativo sobre a fluidodinâmica do leito seco e na evaporação de água do que o ângulo de cone. No entanto, para a secagem do leite desnatado o ângulo de base cônica apresentou interferência significativa.
\end{abstract}

\section{INTRODUÇÃO}

Desde a sua descoberta em 1954 por Gisler e Mathur, o leito de jorro tem sido amplamente estudado, principalmente no que se refere à fluidodinâmica do leito seco e da secagem de pastas. Mathur e Epstein (1974) verificaram que a velocidade mínima de fluidização é dependente tanto das propriedades do sólido e do fluido, bem como da geometria do equipamento. De acordo com Souza (2003) a obtenção do jorro estável também é limitada pela altura máxima de leito, acima desta ocorrem instabilidades no processo. Os parâmetros fluidodinâmicos sofrem ainda, influência das propriedades e dimensões das partículas inertes e da geometria do leito como o ângulo do cone e a relação entre os diâmetros da parte cilíndrica e do orifício de entrada de ar. Olazar et al. (1992) constataram que existem limites de operação para ângulos de cone, para a razão entre o diâmetro de entrada de ar e o diâmetro inferior da base cônica, assim como para a razão entre o diâmetro de entrada de ar e o diâmetro das partículas inertes.

Pham (1983) foi um dos primeiros a verificar que a pasta apresenta influência na fluidodinâmica do leito de jorro. Foi observado que a presença da pasta dificultava a movimentação dos inertes na região anular e também favorecia a formação de regiões estagnadas. Medeiros (2001) investigou a influência da composição química das pastas, utilizando para isso polpas de frutas tropicais. Foi constatado que a presença da pasta com altas concentrações de açúcar causa problema de instabilidades no jorro, enquanto que a presença de gordura e de amido favorece a circulação das 
partículas no leito. Recentemente, Nascimento et al. (2013) estudou o efeito de diferentes concentrações de gordura no leite, durante a secagem desse material em leito de jorro. Foi observado que a ausência de gordura no leite desnatado cauda mudanças significativas na movimentação dos inertes e um aumento expressivo dos valores de queda de pressão. A análise dos trabalhos apresentados mostrou que ainda existe uma escassez de informações na literatura sobre a secagem de pastas em leito de jorro utilizando diferentes configurações de leito. Já que a maioria dos trabalhos avalia o efeito da geometria do leito na sua fluidinâmica, porém sem a presença de pasta. Desta forma, o objetivo deste trabalho foi avaliar o efeito da geometria do leito na secagem de pastas, verificando desta forma se modificando o ângulo de cone é possível melhor os problemas observados por Nascimento et al. (2013) durante a secagem de leite desnatado em leito de jorro.

\section{MATERIAIS E MÉTODOS}

\subsection{Unidade Experimental}

O leito de jorro utilizado neste trabalho é composto por uma coluna cilíndrica de $120 \mathrm{~cm}$ de altura, $30 \mathrm{~cm}$ de diâmetro, diâmetro de entrada de $3 \mathrm{~cm}$ e ângulos da base cônica de 45,60 e $75^{\circ}$. A unidade experimental utilizada e seus respectivos periféricos estão apresentados na Figura 1. Um bocal de entrada de ar do tipo Venturi foi empregado, cujas dimensões estão apresentadas na Figura 2.

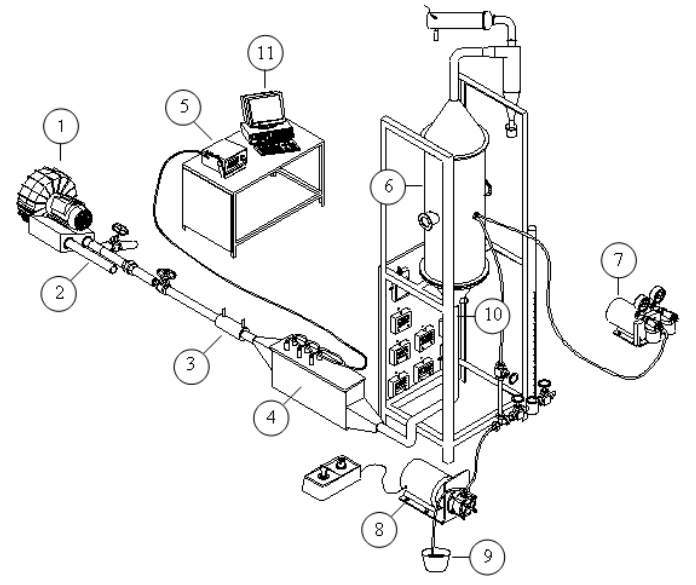

Figura 1 - Unidade experimental: soprador (1); sistema by pass (2); medidor de vazão tipo Venturi (3); trocador de calor (4); controlador de temperatura (5); câmara de secagem (6); compressor de ar (7); bomba peristáltica

(8); reservatório de pasta (9); bocal de entrada de ar

(10) e um sistema de aquisição de dados (11).

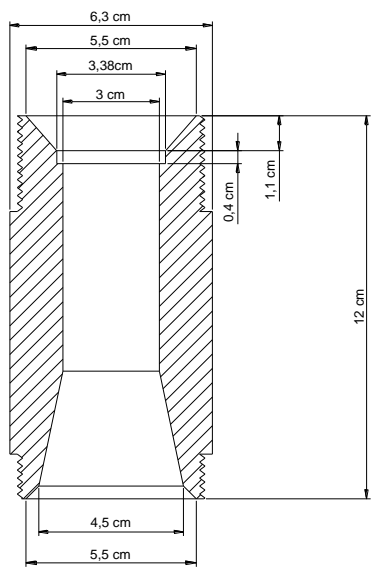

Figura 2 - Bocal de entrada de ar.

Como material inerte foi utilizado partículas de polietileno com 4,38 $\mathrm{mm}$ de diâmetro e massa específica de $930,5 \pm 0,3 \mathrm{~kg} / \mathrm{m}^{3}$. O valor da massa de material inerte utilizado durante o procedimento experimental foi fixado em 1,5; 3,0 e 4,5 kg para todos os valores de ângulo de base cônica avaliados. Água destilada foi utilizada como pasta padrão e leite desnatado como pasta real. Este material foi 
selecionado devido ao fato de apresentar altos valores de queda de pressão e dificuldade de movimentação dos inertes durante a secagem, conforme relatado por Almeida et al. (2010) e Nascimento et al. (2013). A temperatura e a velocidade do ar de entrada foram $100^{\circ} \mathrm{C} \mathrm{e} 1,3 \mathrm{u}_{\mathrm{mj}}$, respectivamente.

\subsection{Procedimento Experimental}

O procedimento experimental foi conduzido em três etapas. Inicialmente foram realizados os ensaios fluidodinâmicos conforme a metodologia proposta por Mathur e Epstein (1974). Este procedimento permitiu a obtenção dos valores de velocidade mínima de jorro e queda de pressão para cada condição experimental avaliada. Com base nos dados obtidos na caracterização fluidodinâmica foram conduzidos os experimentos de evaporação de água. Estes permitiram um conhecimento prévio do comportamento do leito com a presença de uma pasta e determinaram a capacidade máxima de evaporação de água para o leito de jorro utilizado. De posse destes dados os experimentos de secagem do leite desnatado foram realizados. Durante o procedimento experimental foi realizada a obtenção dos valores de velocidade de entrada do ar, de queda de pressão, da temperatura do ar de entrada e de saída, de bulbo seco e úmido na saída do leito em um intervalo de 30 em 30 segundos pelo sistema de aquisição de dados, utilizando uma rotina em labVIEW que fornecia a média e os desvios padrões dos dados obtidos.

\section{RESULTADOS E DISCUSSÕES}

\subsection{Fluidodinâmica do Leito Seco}

A caracterização fluidodinâmica do leite seco permitiu a determinação dos valores de velocidade mínima de jorro, queda de pressão máxima e de jorro estável para as condições de $100^{\circ} \mathrm{C}$, ângulos de cone de 45, 60 e $75^{\circ}$ e massa de material inerte correspondente a 1,5; 3,0 e 4,5 kg. A Tabela 1 apresenta os dados fluidodinâmicos do leito seco obtidos durante os ensaios experimentais.

Tabela 1 - Dados fluidodinâmicos do leito seco obtidos experimentalmente.

\begin{tabular}{|c|c|c|c|c|}
\hline$\theta\left(^{\circ}\right)$ & $\mathrm{m}_{\mathrm{p}}(\mathrm{kg})$ & $\mathrm{u}_{\mathrm{mj}}(\mathrm{m} / \mathrm{s})$ & $\Delta \mathrm{P}(\mathrm{Pa})$ & $\Delta \mathrm{P}_{\max }(\mathrm{Pa})$ \\
\hline 75 & 1,50 & 21,18 & 258,86 & 635,55 \\
\hline 60 & 1,50 & 22,23 & 336,18 & 681,36 \\
\hline 45 & 1,50 & 24,60 & 388,71 & 741,11 \\
\hline 75 & 3,00 & 28,47 & 317,56 & 828,52 \\
\hline 60 & 3,00 & 30,72 & 340,05 & 983,51 \\
\hline 45 & 3,00 & 34,63 & 577,23 & 1512,48 \\
\hline 75 & 4,50 & 35,77 & 378,33 & 1194,70 \\
\hline 60 & 4,50 & 39,72 & 368,57 & 1352,25 \\
\hline 45 & 4,50 & 42,01 & 609,36 & 1754,47 \\
\hline
\end{tabular}

Com base nos dados apresentados na Tabela 1, verificou-se que tanto os valores de velocidade mínima de jorro, $\mathrm{u}_{\mathrm{mj}}$, quanto os de queda de pressão no leito seja ela máxima, $\Delta \mathrm{P}_{\max }$, ou de jorro estável, $\Delta \mathrm{P}$, foram influenciados tanto pela massa de material inerte, $\mathrm{m}_{\mathrm{p}}$, como pelo ângulo de cone, $\theta$. 


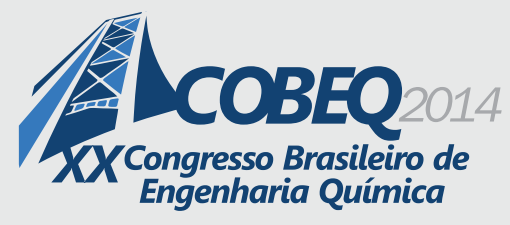

19 a 22 de outubro de 2014
Florianópolis/SC

Os valores dos parâmetros fluidodinâmicos mais elevados foram obtidos para ângulo de $45^{\circ}$. Isto por que a diminuição do ângulo do cone para um valor de $\mathrm{m}_{\mathrm{p}}$ fixo, promove um aumento da altura de leito estático, $\mathrm{H}$, o que consequentemente , proporciona maior resistência ao escoamento de ar e os valores de $\mathrm{u}_{\mathrm{mj}}, \Delta \mathrm{P}$ e $\Delta \mathrm{P}_{\max }$ são desta forma elevados. Referindo-se aos valores de $\Delta \mathrm{P}_{\max }$, resultados similares também foram obtidos por Mathur e Epstein (1974). De acordo com Bi (2011) o mesmo comportamento também foi previsto pelas correlações de Gelperin et al. (1961), Manurung (1964), Kmiec (1980) e Olazar et al. (1993).

Com relação à influência do ângulo do cone na velocidade mínima de jorro, Mathur e Epstein (1974) avaliaram diferentes dados experimentais fornecidos pela literatura utilizando a correlação de Mathur e Gisler. Foi constatado que nos trabalhos que utilizaram leitos de jorro com diâmetro de coluna, $D_{c}$, acima de $30,5 \mathrm{~cm}$ o ângulo de cone não apresentou interferência significativa na velocidade mínima de jorro. Entretanto, foi verificado que para $D_{c}$ igual a $60,1 \mathrm{~cm}$ de diâmetro a velocidade mínima de jorro foi $10 \%$ maior para o ângulo de $85^{\circ}$ quando comparado com o de $45^{\circ}$. Para leitos de jorro cônicos, Olazar et al. (2011) relataram que Dc não precisa ser inserido nas correlações que preveem os valores de $u_{m j}$ ( velocidade mínima de jorro), pois esta não se altera com a variação de $D_{c}$ já que o leito permanece inteiramente na seção cônica. Foi relatado ainda que algumas correlações não utilizam $\theta$, devido ao fato de não ter sido observada influência para a faixa de $\theta$ avaliada ou simplesmente por utilizarem em suas pesquisas apenas um valor de ângulo de cone.

O aumento da massa de material inerte elevou os valores de velocidade mínima de jorro e queda de pressão do leito, como era esperado. Comportamento semelhante foi observado por Mathur e Epstein (1974), Wang et al. (2004), Zhong et al. (2006) e Bi (2011) e Bitti (2012). Os dados experimentais apresentados na Tabela 1 mostram ainda que a massa de material inerte apresentou efeito mais pronunciado do que o ângulo do cone em todos os parâmetros fluidodinâmicos do leito seco analisados.

Existem trabalhos na literatura que avaliam o efeito de $\theta$ para uma altura fixa de leito, não sendo possível desta forma, confrontá-los com os dados experimentais discutidos até o momento. Desta forma, foram realizados experimentos para uma altura fixa de leito correspondente a 20,50 cm, selecionada em testes, para diferentes ângulos de cone, conforme apresentado na Tabela 2.

Tabela 2 - Dados fluidodinâmicos do leito seco obtidos experimentalmente.

\begin{tabular}{|c|c|c|c|}
\hline \multicolumn{4}{|c|}{$\mathbf{H}=\mathbf{2 0 , 5 0} \mathbf{~ c m}$} \\
\hline$\theta\left(^{\circ}\right)$ & $\mathrm{m}_{\mathrm{p}}(\mathrm{kg})$ & $\mathrm{u}_{\mathrm{mj}}(\mathrm{m} / \mathrm{s})$ & $\Delta \mathrm{P}_{\text {máx }}(\mathrm{Pa})$ \\
\hline 75 & 3,84 & 30,42 & 1047,99 \\
\hline 60 & 3,00 & 30,72 & 983,51 \\
\hline 45 & 1,64 & 26,10 & 745,46 \\
\hline
\end{tabular}

Os resultados apresentados na Tabela 2 mostraram que para uma altura fixa de leito à medida que se diminui $\theta$ os valores da queda de pressão máxima do leito também são reduzidos. Este comportamento pode ter ocorrido devido ao fato de que para um valor de $\mathrm{H}$ fixo a massa de inertes utilizada para cada ângulo de cone é modificada. Como pode ser constatado na Tabela 2 o valor de $\mathrm{m}_{\mathrm{p}}$ utilizado para os ângulos de 60 e $75^{\circ}$ é quase do dobro do utilizado para o ângulo de $45^{\circ}$. Desta forma, 


\section{9 a 22 de outubro de 2014 \\ Florianópolis/SC}

a resistência do leito diminui ao ser percolado pelo ar e como consequência os valores de $\Delta \mathrm{P}_{\max }$ também são reduzidos. De acordo com Bi (2011) a correlação de Mukhlenov-Gorshtein (1965) também prevê o mesmo comportamento. No entanto, existe divergência entre alguns trabalhos da literatura, Wang et al. (2004) verificaram que o efeito do ângulo do cone nos valores de $\Delta \mathrm{P}_{\max }$ para uma altura fixa de leito não foi conclusivo. Já Bi (2011) relata que as correlações de Gelperin (1961) e Olazar et al. (1993) preveem comportamento oposto aos obtidos neste trabalho.

Os valores da velocidade mínima de jorro obtidos para os ângulos de 60 e $75^{\circ}$, foram bem próximos conforme mostra a tabela 2 . No entanto, houve uma diminuição desta variável para o ângulo de $45^{\circ}$. Assim como discutido para a queda de pressão, este comportamento pode estar relacionado com a massa de inertes no interior do leito, a qual foi modificada para cada ângulo de cone com o intuito de manter a mesma altura de leito. Olazar et al. (1992) constataram que o efeito do ângulo do cone sobre os parâmetros fluidodinâmicos é mais significativo para alturas mais elevadas de leito estático. Wang et al. (2004) relatam que para alturas de leito estáticos menores que $10 \mathrm{~cm}$ o efeito do ângulo do cone na $u_{m j}$ é reduzido. Olazar et al. (1992) realizaram um estudo avaliando diferentes características de sólidos, geometrias de bocal de entrada de ar distintas e diversos valores de velocidade do ar de entrada. Eles constataram que o diâmetro das partículas inertes exerce grande efeito sobre o comportamento fluidodinâmico do leito. Estas informações mostram que diversos fatores podem exercer interferência conjunta com as demais variáveis avaliadas como, por exemplo, o ângulo do cone que, consequentemente, influência a massa de inerte utilizada para preencher o leito a uma altura fixa, bem como as propriedades do material inerte utilizado.

\subsection{Evaporação de Água e Secagem do Leite Desnatado}

Com base nos resultados obtidos durantes a caracterização do leito de jorro seco, foram realizados os experimentos de evaporação de água. A capacidade máxima de evaporação de água destilada e do leite desnatado é apresentada na Tabela 2.

Tabela 2 - Vazão máxima de alimentação de água destilada e leite desnatado alcançado pelo leito de jorro a $100^{\circ} \mathrm{C}$.

\begin{tabular}{|c|c|c|c|}
\hline$\theta\left(^{\circ}\right)$ & $\mathrm{m}_{\mathrm{p}}(\mathrm{kg})$ & $\mathrm{Q}_{\text {agua }}(\mathrm{mL} / \mathrm{min})$ & $\mathrm{Q}_{\mathrm{ld}}(\mathrm{mL} / \mathrm{min})$ \\
\hline 75 & 3,00 & 35 & - \\
\hline 60 & 3,00 & 40 & - \\
\hline 45 & 3,00 & 40 & - \\
\hline 75 & 4,50 & 45 & 35 \\
\hline 60 & 4,50 & 50 & 30 \\
\hline 45 & 4,50 & 50 & 40 \\
\hline
\end{tabular}

Assim como discutido anteriormente para a fluidodinâmica do leito seco, a massa de material inerte também apresentou maior efeito sobre a taxa de evaporação de água, Qágua, quando comparado ao ângulo do cone, $\theta$. Com base nos resultados apresentados na Tabela 2 observou-se que a única variação de $Q_{\text {água }}$ obtida em função de $\theta$ foi de $5 \mathrm{ml} / \mathrm{min}$ entre os ângulos de 60 e $75^{\circ}$ para todos valores de $\mathrm{m}_{\mathrm{p}}$. $\mathrm{O}$ maior valor $\mathrm{Q}_{\text {água }}$ foi alcançado empregando-se $4,5 \mathrm{~kg}$ de inerte para todos os ângulos de cone avaliados. Desta forma, os experimentos de secagem do leite desnatado foram realizados para 


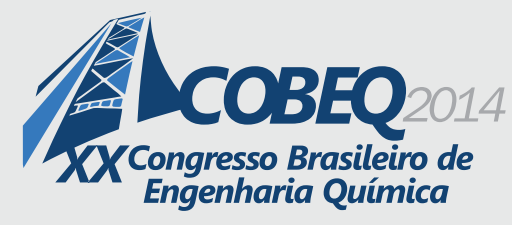

19 a 22 de outubro de 2014
Florianópolis/SC

estas condições de operação, conforme mostra a Tabela 2. Almeida et al. (2010) relata que $\mathrm{m}_{\mathrm{p}}$ interfere fortemente na Qágua. Isto por que um aumento da massa de material inerte eleva a área específica do leito e como a evaporação ocorre na superfície das partículas inertes um aumento da taxa de evaporação de água é obtido. Rodrigues (1993) verificou que a taxa de evaporação de água aumentou à medida que foram variados os ângulos de cone de 60 para $30^{\circ}$. Porém, esta informação não pode ser confrontada com os resultados apresentados na Tabela 2, uma vez que o autor utiliza valores fixos de $\mathrm{H}$.

Os resultados referentes à secagem do leite desnatado mostraram que diferentemente dos dados experimentais apresentados para a água destilada, $\theta$ apresentou interferência nos valores de vazão de alimentação do leite desnatado. Porém, os resultados obtidos não apresentaram nenhuma tendência $\operatorname{com} \theta$, visto que o ângulo de base cônica com o pior desempenho foi o de $60^{\circ}$ e o que obteve maior valor de $\mathrm{Q}_{\mathrm{ld}}$ foi o de $45^{\circ}$. Estes resultados experimentais podem ser melhor compreendidos quando são analisadas as Figuras 6a, 6b e 6c.
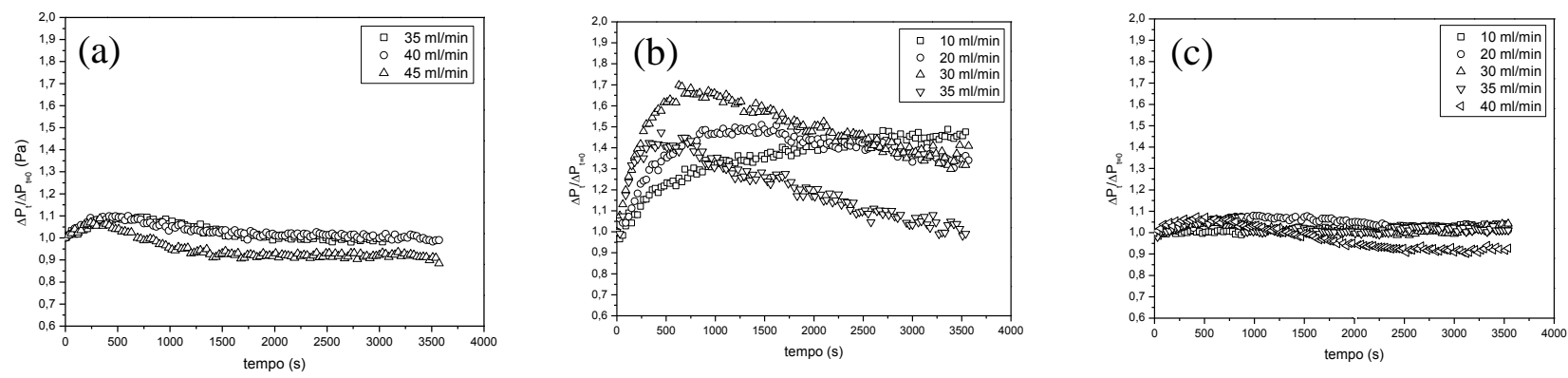

Figura 6 - Adimensional da queda de pressão do leito em função do tempo: (a) ângulo de $45^{\circ}$, (b) ângulo de $60^{\circ}$ e (c) ângulo de $75^{\circ}$.

Os resultados apresentados na Figura $6 \mathrm{~b}$ revelaram que os valores do adimensional de queda de pressão para o ângulo de $60^{\circ}$ foram elevados desviando-se de $\Delta \mathrm{P}_{\mathrm{t}} / \Delta_{\mathrm{t}=0}=1$. Este comportamento já era esperado, visto que a motivação para a escolha da utilização do leite desnatado como pasta foi devido à observação deste desempenho anteriormente por Almeida et al. (2010) e Nascimento et al. (2013) em que também utilizaram como pasta o leite desnatado. Ao analisar as Figuras $6 \mathrm{a}$ e $6 \mathrm{c}$ é possível constatar que os valores do adimensional de queda de pressão para os ângulos de 45 e $75^{\circ}$ foram menos influenciados pela presença da pasta $\left(\Delta \mathrm{P}_{\mathrm{t}} / \Delta_{\mathrm{t}=0}\right.$ próximos de 1$)$ do que os obtidos para o ângulo de $60^{\circ}$. Estes resultados mostram que a variação do ângulo apresentou uma melhora significativa do adimensional da queda de pressão, o que indica que para as condições de operação avaliadas os ângulos de 45 e $75^{\circ}$ são mais indicados para a secagem do leite desnatado em leito de jorro. Comportamento semelhante aos obtidos nas Figuras 6a e 6c também foram observados utilizando água nos trabalhos de Almeida et al. (2010) e Bitti (2012). Isto mostra que para os ângulos de 45 e $75^{\circ}$ o leito de jorro não "sentiu" significativamente a presença da pasta. Ayub (1993) estudou o recobrimento de comprimidos em um leito de jorro com ângulos de base cônica de 30,45 e $60^{\circ}$. $\mathrm{O}$ autor constatou que o ângulo de $45^{\circ}$ apresentou melhor circulação dos comprimidos, o que favoreceu a um recobrimento mais uniforme, e as altas taxas de crescimento e eficiência do recobrimento. Foi constatado ainda que o ângulo de $60^{\circ}$ também apresentou boa movimentação das partículas, no 
entanto, essa movimentação dos comprimidos gerou um pequeno desgaste e pontos de quebra nas arestas dos comprimidos. O autor constatou ainda que quanto menor a esfericidade da partícula maior deve ser a inclinação da base do leito. Esta ultima observação confirma o que foi discutido anteriormente para fluidodinâmica do leito seco, fatores como o tipo de material inerte também podem contribuir para uma interferência conjunta, por exemplo, com o ângulo do cone. Com relação às informações sobre a secagem de pastas utilizando diferentes configurações de leito, verificou-se ainda a existência de poucos trabalhos sobre este assunto. Assim como esperado, os ensaios de secagem do leite desnatado mostram ainda que a pasta exerceu influência durante o processo de secagem, já que os valores obtidos foram inferiores aos alcançados utilizando água destilada. Uma melhor discussão sobre este assunto pode ser encontrada nos trabalhos de Pham (1983), Medeiros (2001), Almeida et al. (2010) e Nascimento et al. (2013).

\section{CONCLUSÕES}

Neste trabalho foi realizada uma avaliação do efeito do ângulo de base cônica e da massa de material inerte na fluidodinâmica do leito seco e na secagem de leite desnatado em leito de jorro. Os resultados experimentais mostraram que tanto os parâmetros fluidodinâmicos do leito seco quanto à evaporação de água foram influenciados tanto pela massa de material inerte e como pelo ângulo do cone estudado. No entanto, verificou-se que a massa de material inerte apresentou maior interferência. Com relação à secagem do leite desnatado, constatou-se que o ângulo de cone influenciou fortemente o adimensional de queda de pressão e que o ângulo de $60^{\circ}$ apresentou, do ponto de vista da variação da pressão, o pior desempenho para a secagem deste tipo de pasta em leito de jorro.

\section{NOMENCLATURA}

$\mathrm{D}_{\mathrm{c}} \quad$ diâmetro da coluna

$\mathrm{H} \quad$ altura de leito estático

[cm]

$\mathrm{m}_{\mathrm{p}} \quad$ massa de partículas inertes

[cm]

$\mathrm{P} \quad$ pressão de jorro estável

$[\mathrm{kg}]$

$[\mathrm{Pa}]$

$\mathrm{P}_{\max } \quad$ pressão máxima

$[\mathrm{Pa}]$

Q agua $\quad$ vazão de alimentação de água destilada

[mL/min]

$Q_{\text {ld }}$

vazão de alimentação de leite desnatado

$[\mathrm{mL} / \mathrm{min}]$

$\mathrm{t}$ tempo

[s]

$\mathrm{u}_{\mathrm{mj}} \quad$ velocidade mínima de jorro

[m/s]

$\theta \quad$ ângulo da base cônica

[-]

$\Delta$

diferença

$[-]$

\section{REFERÊNCIAS}

Almeida, A. R. F.; Freire, F. B.; Freire, J. T. Transient analysis of pasty material drying in a spouted bed of inert particles. Drying Technol., v.28 (3), p. 330-340, 2010.

Ayub, G. S. E. Recobrimento de comprimidos em leito de jorro bi-dimensional: análise do crescimento e transferência de calor gás-partícula. Campinas, 1993. 107 p. Dissertação (Mestrado 
em Engenharia Química) Faculdade de Engenharia Química, Universidade Estadual de Campinas, 1993.

Bi X. Initiation of spouting. In: Epstein, N.; Grace, J. R. Spouted and Spouted-Fluid Beds: Fundamentals and Applications. New York: Cambridge University Press Co., 2011. p. 17-28.

Bitti, M. T. Avaliação das condições operacionais da taxa de evaporação de água em leito de jorro. São Carlos, 2012. 59 p. Dissertação (Mestrado em Engenharia Química), Universidade Federal de São Carlos, 2012.

Himmelblau, D. M. Accounts of Experiences in the Application of Artificial Neural Networks in Chemical Engineering. Ind. Eng. Chem. Res., v. 47, p. 5782-5796, 2008.

Mathur, K. B.; Epstein, N. Spouted Beds. New York: Academic Press, 1974.

Medeiros, M. F. D. Influência da composição química dos materiais no desempenho do processo de secagem de polpas de frutas em leito de jorro. Campinas, 2001. 247 p. Tese (Doutorado em Engenharia Química) Faculdade de Engenharia Química, Universidade Estadual de Campinas, 2001.

Nascimento, B. S.; Freire, F. B.; Freire, J. T. Moisture prediction during paste drying in a spouted bed. Drying Technol., v. 31(15), p. 1808-1816, 2013.

Olazar, M.; San José, M. J.; Aguayo, A. T.; Arandes, J. M; Bilbao, J. Stable operation conditions for gas-solid contact regimes in conical spouted beds. Ind. Eng. Chem. Res., v. 31 (7), p. 1784-1792, 1992.

Olazar M.; San José, M. J.; Bilbao, J. Conical spouted beds. Conical spouted beds. In: Epstein, N.; Grace, J. R. Spouted and Spouted-Fluid Beds: Fundamentals and Applications. New York: Cambridge University Press Co., 2011.p. 82 - 104.

Pham, Q. T. Behavior of a conical spouted-bed dryer for animal blood. Can. J. Chem. Eng., v. 61, p. 426-434, 1983.

Rodrigues, C. C. Análise da secagem de suspensões em leito de jorro com partículas inertes. São Carlos, 1993. 116 p. Dissertação (Mestrado em Engenharia Química), Universidade Federal de São Carlos, 1993.

Souza, C. R. F. Estudo comparativo da produção de extrato seco de Bauhinia forficata Link pelos processos spray-dryer e leito de jorro. Ribeirão Preto, 2003. Dissertação (Mestrado em Ciências Farmacêuticas) Faculdade de Ciências Farmacêuticas de Ribeirão Preto, Universidade de São Paulo, 2003.

Wang, Z.; Bi, H. T., Lim, C. J. Su, P. Determination of minimum spouting velocities in conical spouted beds. Can. J. Chem. Eng., v. 82, p. 11-19, 2004.

Zhong, W.; Chen, X. Zhang, M. Hydrodynamic characteristics of spout-fluid bed: Pressure drop and minimum spouting/spout-fluidizing velocity. Chem. Eng. J., v. 118, p. 37-46, 2006. 